\title{
Treatment of Periprosthetic Joint Infection in Total Knee Arthroplasty with a Temporary Intramedullary Nail: Is a Long or Short Nail Better?
}

\author{
Nequesha S. Mohamed, MD ${ }^{1}$ Iciar M. Davila Castrodad, MD ${ }^{2}$ Jennifer I. Etcheson, MD, MS 3 \\ Margaret N. Kelemen ${ }^{4} \quad$ F. Johannes Plate, MD, $\mathrm{PhD}^{1}$ Janet D. Conway, MD ${ }^{4}$ Ronald E. Delanois, MD ${ }^{4(0)}$ \\ ${ }^{1}$ Department of Orthopedic Surgery, Wake Forest School of Medicine, \\ Winston-Salem, North Carolina \\ 2 Department of Orthopedic Surgery, Hackensack Meridian School of \\ Medicine, Seton Hall University, Nutley, New Jersey \\ ${ }^{3}$ Department of Orthopedic Surgery and Rehabilitation Medicine, \\ SUNY Downstate Medical Center, Brooklyn, New York \\ ${ }^{4}$ Center for Joint Preservation and Replacement, Rubin Institute for \\ Advanced Orthopedics, Sinai Hospital of Baltimore, Baltimore, \\ Maryland
Address for correspondence Ronald E. Delanois, MD, Center for Joint Preservation and Replacement, Rubin Institute for Advanced Orthopedics, Sinai Hospital of Baltimore, 2401 West Belvedere Avenue, Baltimore, MD 21215 (e-mail: delanois@me.com).

J Knee Surg 2023;36:39-46.

\section{Abstract \\ Keywords \\ - periprosthetic joint infection \\ - total knee arthroplasty \\ - intramedullary nail \\ - two-stage exchange arthroplasty \\ - revision total knee arthroplasty}

To our knowledge, no studies have compared postoperative outcomes between patients who received a temporary short or long intramedullary (IM) nail in the setting of infected total knee arthroplasty (TKA). Therefore, the aim of this study was to compare short-term outcomes for patients who underwent long or short IM nail insertion for treatment of periprosthetic knee infection. Specifically, we compared: (1) success rates; (2) patient reported/functional outcomes; and (3) complications between patients implanted with a short or a long IM nail following PJI of the knee. A retrospective chart review was performed for patients who underwent two-stage exchange arthroplasty with a temporary long or short IM nail between November 2010 and June 2018 at our institution $(n=67)$. Continuous and categorical variables were assessed using $t$-test/Mann-Whitney $U$ test and chi-squared test, respectively. Logistic regression analyses were conducted to assess the effect of IM nail length on success rate while adjusting for age, sex, body mass index, and race. A total of 36 patients underwent temporary treatment with a long IM nail, while 31 patients received a short IM nail. There were no differences in success rate for reimplanted patients treated with long and short IM nails (odds ratio 0.992; $p=0.847$ ). Fewer patients with a long IM nail went on to reimplantation ( 52.8 vs. $83.9 \% ; p=0.007$ ). There was no difference in satisfaction ( 7.86 vs. $7.68 ; p=0.515$ ), pain scores ( 3.39 vs. 4.45 points; $p=0.126$ ), or Knee Society score outcome scores ( 150.61 vs. 166.26 points; $p=0.117$ ) between long or short IM nail patients. Following reimplantation, there was no difference in the number of patients who became reinfected ( 15.8 vs. $11.5 \% ; p=0.679)$ or went on to amputation ( 0 vs. $7.7 \% ; p=0.210$ ). Periprosthetic joint infection (PJI) is a rare but received

January 27, 2021

accepted

March 12, 2021

article published online

May 4, 2021 (c) 2021. Thieme. All rights reserved.

Thieme Medical Publishers, Inc., 333 Seventh Avenue, 18th Floor, New York, NY 10001, USA
DOI https://doi.org/

10.1055/s-0041-1729552. ISSN 1538-8506. 
serious postoperative complication following TKA. Our findings suggest that the use of long and short IM nails during two-stage exchange can have equal utility in PJI patients with severe bone defects.

Periprosthetic joint infection (PJI) after total knee arthroplasty (TKA) is an uncommon but severe complication associated with poor patient outcomes and high expenditures. Although less than $3 \%$ of primary TKA patients may develop an infection, up to $23 \%$ of revision TKAs are complicated by a subsequent infection. $^{1-4}$ The preferred method of treatment for PJI, twostage exchange revision arthroplasty, has a success rate of up to $95 \%{ }^{5-7}$ However, its success decreases with each subsequent revision patients undergo, as multiple revisions can lead to significant bone loss and compromised host status. ${ }^{5,8-10}$ Undergoing revision with traditional antibiotic spacers has proven reliable with adequate eradication rates and functional outcomes, but spacer utilization can lead to bone loss and increased risk of spacer displacement. ${ }^{11-17}$ Therefore, other stabilization and infection eradication techniques have been explored in this cohort of patients.

As a form of static immobilization, intramedullary (IM) nailing have been utilized as an alternative to conventional spacers during two-stage revision. This technique can deliver antibiotics locally and offer sufficient knee stability for patients with preexisting bone loss to allow immediate postoperative ambulation. ${ }^{18,19}$ In the setting of infected TKA, IM nails have demonstrated appropriate efficacy, particularly in patients with extensive bone loss. ${ }^{20-22}$ Specifically, Waldman et al demonstrated that $100 \%$ of TKA patients with PJI became infection free after treatment with an IM nail. ${ }^{20}$ Similarly, Mohamed et al reported a $74.2 \%$ successful treatment rate in repeatedly revised patients with bone loss implanted with a temporary short IM nail. ${ }^{22}$ Others have reported positive outcomes with infection-free success rates ranging from 86.5 to $92 \% .^{21,23,24}$ Despite the evidence supporting temporary IM nailing for PJI following TKA, the type of nail that can provide optimal outcomes has not been well defined.

Our institution has utilized both short and long IM nails to treat repeat PJI patients with Anderson Orthopaedic Research Institute (AORI) Type II or III bone loss. To date, no studies have compared the postoperative outcomes of these nails when used in this population to treat PJI during a twostage revision. Therefore, the aim of this study was to compare short-term postoperative outcomes for patients who underwent temporary long or short IM nail insertion for treatment of PJI. Specifically, we compared: (1) success rates; (2) patient reported and functional outcomes; and (3) complications between patients implanted with a short or a long IM nail following PJI of the knee.

\section{Materials and Methods}

\section{Patient Selection}

A retrospective chart review was performed for all patients with repeat periprosthetic knee infections at our institution. All patients with Type II or III bone defects, as classified according to the AORI system, ${ }^{25}$ who underwent two-stage exchange arthroplasty with a temporary long or short IM nail between March 1, 2010, and June 30, 2018, were included for analysis. Patients with AORI Type 0 or I bone defects, PJI treated with antibiotic suppression, debridement with prosthesis retention, and resection arthroplasty were excluded. Patients with prior operations were not excluded. Demographic factors such as age, sex, body mass index (BMI), race, and health status, were collected for all patients. Our institutional review board determined this project exempt from review as it did not meet the criteria for human subjects research.

A total of 389 patients with an infected TKA were identified between 2009 and 2015. Of this total, 167 patients were treated with antibiotic suppression, irrigation and debridement (I\&D), or resection arthroplasty. Of the resulting 222 patients, 155 had no preexisting bone loss and underwent two-stage exchange with articulating or nonarticulating spacers. Thus, a total of 67 patients, 31 men and 36 women, were included, 36 long IM nail patients and 31 short IM nail patients. The patients included in the short nail cohort are part of a previously published study. ${ }^{22}$ There were no significant differences in mean ages (63 vs. 62 years; $p=0.746$ ), sex ( 55.6 vs. $44.4 \%$ females; $p=0.193$ ), race (55.6 vs. $61.3 \%$ whites; $p=0.635$ ), or mean follow-up (25.4 vs. 26.0 months; $p=0.486$ ) between the long and short IM nail cohorts. Mean BMI was the only patient demographic that significantly differed between the long and short IM nail groups (39.8 vs. $31.8 \mathrm{~kg} / \mathrm{m}^{2} ; p<0.001$ ) (-Table 1). Regarding health status, we found no difference in mean serum albumin levels ( $2.9 \mathrm{vs}$. $3.1 ; p=0.283$ ) or McPherson et al's systemic host grade $(p=0.821)$ between the long and short IM nail cohorts (-Table 2). Additionally, there was no difference in the organism cultured at initial IM nail placement $(p=0.105)$

(-Table 3).

\section{Management Technique}

Surgery consisted of removal of the infected prostheses, I\&D, and IM nail insertion. The IM nails were selected based on surgeon preference and were coated with antibiotic cement. The cement was composed of liquid monomer, $3.6 \mathrm{~g}$ of tobramycin, and $1 \mathrm{~g}$ of vancomycin per every $40 \mathrm{-g}$ package of cement prior to insertion. Tobramycin antibiotic powder ( $4.6 \mathrm{~g}$ ) alone was used if a patient had an allergy to vancomycin. All patients received standardized institutional postoperative care and were instructed to weight-bear as tolerated immediately. Parenteral antibiotics were prescribed for at least 6 weeks under the guidance of blood and aspirate cultures as well as microbiome sensitivities. Antibiotics were prolonged in cases where infection was not eradicated. Serum infection markers, such as C-reactive protein (CRP) and erythrocyte sedimentation rate (ESR), were assessed throughout the course of antibiotic therapy. 
Table 1 Descriptive analysis demonstrating patient demographics

\begin{tabular}{|l|l|l|l|}
\hline & Long intramedullary nail & Short intramedullary nail & $p$-Value \\
\hline Number of patients & 36 & 31 & \\
\hline Age $^{\mathrm{a}}(\mathrm{y})$ & $63.4(9.8)$ & $62.4(13.8)$ & 0.746 \\
\hline $\mathrm{BMI}^{\mathrm{a}}\left(\mathrm{kg} / \mathrm{m}^{2}\right)$ & $39.8(8.1)$ & $31.8(7.0)$ & $<0.001$ \\
\hline Length of stay $^{\mathrm{a}}(\mathrm{d})$ & $7.4(7.8)$ & $5.8(3.2)$ & 0.278 \\
\hline Female & $20(55.6 \%)$ & $16(44.4 \%)$ & 0.193 \\
\hline Race & \multicolumn{3}{|l}{} \\
\hline White & $20(55.6 \%)$ & $19(61.3 \%)$ & 0.635 \\
\hline African American & $16(44.4 \%)$ & $12(38.7 \%)$ & \\
\hline
\end{tabular}

Abbreviation: BMI, body mass index.

${ }^{a}$ Values are reported as mean and standard deviation in parentheses.

Table 2 Host factors

\begin{tabular}{|l|l|l|l|}
\hline & Long intramedullary nail & Short intramedullary nail & $p$-Value \\
\hline Albumin $^{\text {a }}$ & $2.9(0.6)$ & $3.1(0.7)$ & 0.283 \\
\hline McPherson et al's systemic host grade & $4(12.9 \%)$ & 0.821 \\
\hline A & $3(8.3 \%)$ & $20(64.5 \%)$ & \\
\hline B & $25(69.4 \%)$ & $7(22.6 \%)$ & \\
\hline C & $8(22.2 \%)$ & & \\
\hline
\end{tabular}

${ }^{\mathrm{a}}$ Reported as mean and standard deviation in parentheses.

In cases where the CRP and ESR levels were questionable, joint aspiration was performed to rule out ongoing infection. Once patients were considered to have cleared the infection by improvement in symptoms, downward trend- ing in CRP and ESR levels, or a negative aspiration, they were scheduled for reimplantation. Patients were reimplanted if intraoperative pathology reported negative frozen sections.

Table 3 Organism cultured at initial intramedullary nail placement

\begin{tabular}{|l|l|l|l|}
\hline & Long intramedullary nail & Short intramedullary nail & $p$-Value \\
\hline Periprosthetic knee infections $(N)$ & 36 & 31 & 0.105 \\
\hline No growth & $15(41.7 \%)$ & $13(41.9 \%)$ \\
\hline Methicillin-resistant Staphylococcus aureus & $4(11.1 \%)$ & $4(12.9 \%)$ \\
\hline Enterococcus group D & $2(5.6 \%)$ & $1(3.2 \%)$ \\
\hline Vancomycin-resistant enterococci group D & $1(2.8 \%)$ & $1(3.2 \%)$ \\
\hline Pseudomonas aeruginosa & $2(5.6 \%)$ & $0(0 \%)$ \\
\hline Staphylococcus aureus & $4(11.1 \%)$ & $0(0 \%)$ \\
\hline Coagulase-negative staphylococci & $4(11.1 \%)$ & $8(25.8 \%)$ \\
\hline Streptococcus anginosus & $1(2.8 \%)$ & $0(0 \%)$ \\
\hline Streptococcus viridians & $1(2.8 \%)$ & $0(0 \%)$ \\
\hline Candida albicans & $0(0 \%)$ & $1(3.2 \%)$ \\
\hline Escherichia coli & $0(0 \%)$ & $1(3.2 \%)$ \\
\hline Polymicrobial & $1(2.8 \%)$ & $2(6.5 \%)$ \\
\hline Proteus mirabilis & $1(2.8 \%)$ & $0(0 \%)$ \\
\hline
\end{tabular}




\section{Variables}

Health status was graded preoperatively according to patient serum albumin levels and the McPherson et al's classification. ${ }^{26}$ The microorganism cultured from intraoperative tissue specimens was collected and compared between the cohorts. Treatment success was determined using the Delphi-based consensus definition of successfully treated PJI: infection eradication, no further surgical intervention for infection after reimplantation, and no PJI-related mortality. ${ }^{27}$

Postoperative complications such as reinfection and amputation were documented. Patient-reported outcomes included pain and satisfaction. Perioperative pain intensity was measured by utilizing patient visual analog scale (VAS) scores. In addition to preoperative baseline scores, VAS scores were collected up to 48 hours postoperatively at 8hour intervals and reported as area under the curve. Satisfaction was assessed via a postoperative survey, and scored out of 10. Functional outcomes were assessed using VAS and Knee Society scores (KSSs) from the patient's last clinic follow-up visit.

\section{Data Analysis}

Continuous and categorical variables were assessed using Student's $t$-test, Mann-Whitney $U$ test, and chi-squared test, respectively. Logistic regression analyses were conducted to assess the effect of IM nail length on success rate while adjusting for age, sex, BMI, and race. A $p$-value of 0.05 was set as the threshold for statistical significance. All statistical analysis was performed using SPSS version 25 (IBM Corporation; Armonk, NY).

\section{Results}

\section{Success Rate}

After adjusting for age, sex, BMI, and race, there was no differences in success rates for reimplanted patients treated with long and short IM nails (odds ratio 0.992; $p=0.847$ ) at a mean follow-up of 26.0 months ( - Table 4 ). Fewer patients with a long IM nail went on to reimplantation compared with patients with a short IM nail (52.8 vs. $83.9 \% ; p=0.007)$ ( - Table 5). In the interchange period, there were no significant differences between long and short IM nail cohorts requiring exchange for a second nail (31.6 vs. $11.5 \%$; $p=0.137)$.

\section{Patient-Reported and Functional Outcomes}

There were no differences in pain intensity between the long and short IM nail groups during the 48-hour interval postoperatively (114.61 vs. 134.93 through $0-24$ hours; $p=0.148$ and 128.50 vs. 143.74 points through 24-48 hours; $p=0.191$ ) (-Table 6). Similarly, there were no differences reported in postoperative satisfaction (7.86 vs. 7.68; $p=0.515$ ). There were also no differences in VAS pain scores (3.39 vs. 4.45 points; $p=0.126$ ) and KSS functional outcome scores ( 150.61 vs. 166.26 points; $p=0.117$ ) between patients reimplanted with a long or short IM nail at a mean follow-up of 32 months.

\section{Complications}

Following reimplantation, there was no difference in the number of patients who became reinfected (15.8 vs. $11.5 \%$;

Table 4 Logistic regression model for treatment success

\begin{tabular}{|c|c|c|c|}
\hline Variable & Odds ratio & $p$-Value & $95 \%$ confidence interval \\
\hline Age & 0.997 & 0.917 & $0.945-1.053$ \\
\hline BMI & 0.992 & 0.847 & $0.915-1.076$ \\
\hline \multicolumn{4}{|l|}{ Race } \\
\hline Black or African American & - & Reference & - \\
\hline White & 3.354 & 0.033 & $1.101-10.223$ \\
\hline \multicolumn{4}{|l|}{ Sex } \\
\hline Female & - & Reference & - \\
\hline Male & 1.472 & 0.499 & $0.480-4.510$ \\
\hline \multicolumn{4}{|l|}{ Host immune status } \\
\hline A & - & Reference & - \\
\hline$B$ & 1.234 & 0.827 & $0.189-8.071$ \\
\hline C & 0.907 & 0.934 & $0.90-9.156$ \\
\hline Albumin & 1.206 & 0.724 & $0.427-3.402$ \\
\hline Required nail exchange & 0.512 & 0.422 & $0.100-2.619$ \\
\hline \multicolumn{4}{|l|}{ IM nail length } \\
\hline Long & - & Reference & - \\
\hline Short & 0.992 & 0.847 & $0.915-1.076$ \\
\hline
\end{tabular}

Abbreviations: BMI, body mass index; IM, intramedullary. 
Table 5 Outcomes and complications for temporary long and short intramedullary nails

\begin{tabular}{|c|c|c|c|}
\hline & Long intramedullary nail & Short intramedullary nail & $p$-Value \\
\hline Number of periprosthetic knee infections $(N)$ & 36 & 31 & \\
\hline \multicolumn{4}{|l|}{ Outcome measure } \\
\hline Follow-up for alla (mo) & $31.5(24.0-226.0)$ & $32.0(24.2-85.0)$ & 0.806 \\
\hline Duration of antibiotics $^{\mathrm{a}}$ (d) (SD) & $42.0(35-42)$ & $42.0(14-56)$ & 0.457 \\
\hline $\operatorname{LOS}^{b}(d)$ & $7.42(7.8)$ & $5.77(3.2)$ & 0.278 \\
\hline \multicolumn{4}{|l|}{ Reimplantation } \\
\hline Reimplanted & $19(52.8 \%)$ & $26(83.9 \%)$ & 0.007 \\
\hline Time until reimplant $^{\mathrm{a}}(\mathrm{mo})$ & $10.3(4.0-41.0)$ & $2.7(0.1-16)$ & 0.008 \\
\hline Treatment success & $16(84.2 \%)$ & 19 (73.1\%) & 0.481 \\
\hline \multicolumn{4}{|l|}{ Interchange period } \\
\hline Required a second intramedullary nail $^{c}$ & $6(31.6 \%)$ & $3(11.5 \%)$ & 0.137 \\
\hline \multicolumn{4}{|l|}{ Complications following reimplantation } \\
\hline Infection $^{c}$ & $3(15.8 \%)$ & $3(11.5 \%)$ & 0.679 \\
\hline Amputation $^{c}$ & $0(0 \%)$ & $2(7.7 \%)$ & 0.210 \\
\hline \multicolumn{4}{|l|}{ Retention of nail } \\
\hline Retention of nail $^{\mathrm{C}}$ & $17(47.2 \%)$ & $5(16.1 \%)$ & 0.007 \\
\hline Treatment success $^{c}$ & $16(94.1 \%)$ & $4(80.0 \%)$ & 0.334 \\
\hline \multicolumn{4}{|l|}{ Complications with retention of nail } \\
\hline Infection $^{c}$ & $1(5.9 \%)$ & $0(0 \%)$ & 0.999 \\
\hline Amputation $^{\mathrm{c}}$ & $1(5.9 \%)$ & $0(0 \%)$ & 0.999 \\
\hline Irrigation and debridement ${ }^{\mathrm{C}}$ & $0(0 \%)$ & $1(20.0 \%)$ & 0.999 \\
\hline
\end{tabular}

Abbreviations: LOS, length of stay; SD, standard deviation.

${ }^{a}$ Median (range).

${ }^{\mathrm{b}}$ The values are given as the mean and range in parentheses.

'The values are given as the number of cases with the percentage in parentheses. The percentages were determined from the total number of cases that retained the intramedullary nail and cases that went on to reimplantation.

Table 6 Patient-reported outcomes and functional scores for patients treated with temporary long or short intramedullary nails

\begin{tabular}{|l|l|l|l|}
\hline \multicolumn{2}{|l|}{ Long intramedullary nail } & Short intramedullary nail & $p$-Value \\
\hline Pain intensity & $114.61(44)$ & $134.93(65.13)$ & 0.148 \\
\hline Pain intensity (AUC) 0-24h & $128.50(38.21)$ & $143.74(53.37)$ & 0.191 \\
\hline Pain intensity (AUC) 24-48 h & $7.86(1.15)$ & $7.68(1.13)$ & 0.515 \\
\hline Patient-reported satisfaction (out of 10) & $103.52(23.41)$ \\
\hline Satisfaction & $117.61(27.91)$ & $5.29(3.16)$ & 0.198 \\
\hline Functional and PRO scores at last follow-up for patients who retained the IM nail & 0.154 \\
\hline KSS & $4.52(2.63)$ & $7.33(1.03)$ & 0.553 \\
\hline VAS & $7.65(1.11)$ & $166.26(33.72)$ & \\
\hline Satisfaction & $150.61(45.01)$ & $4.45(3.19)$ & 0.117 \\
\hline Functional and PRO scores at last follow-up for reimplanted patients & $7.76(1.16)$ & 0.126 \\
\hline KSS & $3.39(2.23)$ & 0.416 \\
\hline VAS & $8.05(1.18)$ & \\
\hline Satisfaction &
\end{tabular}

Abbreviations: AUC, area under the curve; IM, intramedullary; KSS, Knee Society score; PRO, patient-reported outcomes; VAS, visual analog scale. Note: The values are given as the mean and standard deviation in parentheses. 
$p=0.679$ ) or went on to amputation ( 0 vs. $7.7 \% ; p=0.210$ )

(-Table 5).

\section{Discussion}

PJI following TKA is a serious complication associated with increased patient morbidity and mortality. Temporary IM nail utilization in combination with two-stage exchange arthroplasty is an option in the treatment of PJI when severe bone loss is present, as the nail offers sufficient knee stability for immediate mobilization. ${ }^{18,22}$ Following infection eradication and explantation of the nail, patient function can potentially be restored, and activity levels may improve. Both long and short IM nails have been successful in two-stage exchange, but their outcomes have not been previously compared. Therefore, this study compared short-term postoperative outcomes for patients who underwent long or short IM nail insertion for treatment of PJI during two-stage exchange arthroplasty. Our findings demonstrated fewer long nail patients underwent reimplantation, retaining their nail after surgery. We also found that there were no statistical differences in reinfection, amputation, or successful infection eradication rates between the cohorts. Additionally, no differences in patient-reported or functional outcomes were noted between the cohorts. The utilization of both long and short IM nails during a two-stage revision TKA are comparable in patients with prior bone loss.

This study is not without limitations. We found a difference in BMI between the long and short IM nail cohorts. Long IM nails are implanted in patients with larger stature due to surgeon preference, and therefore, it would be expected to see a higher BMI in this group. Nevertheless, this difference did not affect the statistical analysis as this variable was adjusted for in the logistic regression analysis. Furthermore, as the incidence of PJI is as low as 0.7 to $2 \%,{ }^{28}$ a large number of study patients are necessary to detect significant differences between cohorts. Although this study is likely underpowered to detect this difference, a comparison between long and short IM nails has not been explored, and is therefore valuable, particularly because differing implants can achieve temporary fixation during PJI treatment. Furthermore, post hoc power analysis with study parameters of $80 \%$ power and an $\alpha=0.05$ revealed the need for 68 total patients, which is in line with our study.

Our study found that fewer patients with a long IM nail went on to reimplantation. Per chart review, patients felt satisfied that they had adequate stability and pain control and were reluctant to undergo further surgical intervention. These patients were in effect, successfully managed with a one-stage exchange. Of those who underwent reimplantation, we found varied success rates for infection eradication between the long and short nail cohorts. Similarly, studies regarding nonarticulating cement spacers, which are comparable to IM nails, report wide-ranging infection eradication rates. Fehring et al performed a retrospective study on 25 PJI patients treated with a static cement spacer and found 22 patients ( $88 \%$ ) were free of infection at a mean follow-up of 36 months. ${ }^{16}$ The authors reported unexpected bone loss in $15(60 \%)$ of the 25 patients, a complication commonly associated with the use of these spacers. ${ }^{4,13}$ In another retrospective review of failed infected TKAs, Johnson et al reported a success rate of $83 \%$ ( 28 of 34 patients) in their cohort treated with nonarticulating cement spacers. ${ }^{12}$ Conversely, Choi et al performed a retrospective study on $33 \mathrm{PJI}$ patients treated with static cement spacers and found 22 patients $(67 \%)$ were infection free at 58 months of follow-up. ${ }^{29}$ These studies demonstrate the variability of infection eradication with traditional static spacers, which help corroborate the eradication rates of the IM nails utilized as nontraditional spacers in this study.

We found no differences in postoperative pain intensity or KSSs between the groups at a mean 2-year follow-up. Several studies have shown that functional outcome also improved when patients treated with static spacers undergo reimplantation. Lichstein et al performed a retrospective case series on 107 infected TKA patients and found that KSS knee scores improved from a preoperative median of 36 (range: 24-48) to a postoperative median of 86 (range: 22-45). ${ }^{30}$ They also found that KSS function scores increased from a median of 32 (range: 22-45) to a postoperative median of 85 (range: 61-97). Fehring et al utilized the Hospital for Special Surgery rating to evaluate functional outcomes and found that patients treated with a static spacer block $(n=25)$ had an improved mean score of 83 points (range: $37-98){ }^{16}$ Our study expands on these previous studies by including a pain intensity analysis, which these studies fail to include.

Our findings demonstrated no difference in the number of patients who became reinfected or went on to amputation. In the cohort of previously published short nail patients, Mohamed et al reported the observed overall success rate of $74.1 \%$, with a reinfection rate of $11.5 \%$ and no amputations. ${ }^{22}$ Other studies report similar reinfection rates and subsequent surgical management. A retrospective review by Emerson et al studied postoperative outcomes for 26 patients treated with a static block spacer for infected TKAs. ${ }^{17}$ The authors reported a reinfection rate of $7.6 \%$ at 36 months that increased to $23 \%$ ( 6 of 26) after an average of 7.5 years. In a retrospective study of 38 patients implanted with static spacers, Freeman et al reported three reoperations (7.8\%) in the group of patients who remained infected or became reinfected. ${ }^{31}$ In another retrospective study evaluating outcomes for cement spacers $(n=10)$, Jämsen et al reported that one patient underwent an above-theknee amputation due to a life-threatening infection. ${ }^{32}$ Despite the small sample sizes reported, results from these studies coincide with our findings, reporting low reinfection and amputation rates. Our comparative study demonstrates similar outcomes between IM nails and static spacers, suggesting they have a place in the treatment of infected TKA during two-stage exchange arthroplasty. A long IM nail offers the potential for a one-stage revision, while a short IM nail offers increased mobilization during the interim of a two-stage exchange. This information can provide surgeons with more tools to assist their more complex patients. 


\section{Conclusion}

Temporary IM nail placement can be an effective alternative technique to traditional antibiotic spacers for PJI patients with considerable bone loss. This study compared long and short IM nails in this population of patients, and found a subset of patients implanted with a long IM nail were successfully treated with one-stage exchange. We also found no difference in success rates for infection eradication for reimplanted patients treated with a long or short IM nail at short-term follow-up. These data suggest that the use of long and short IM nails during two-stage exchange can have equal utility in PJI patients with severe bone defects.

Note

Institutional Review Board has determined this project exempt from review as this does not meet the criteria for human subjects research.

\section{Conflict of Interest}

J.D.C. reports personal fees from Biocomposities, personal fees from Bonesupport, personal fees from DePuy Synthes, personal fees from MHE Coalition, personal fees from Orthofix, Inc, personal fees from OrthoPediatrics, personal fees from Pega Medical, personal fees from Smith and Nephew, personal fees from Stryker, personal fees from University of Florida, personal fees from Zimmer, personal fees from Zimmer Biomet, outside the submitted work. $R$. E.D. reports other from Flexion Therapeutics, other from Orthofix Inc, other from Stryker Corp, other from United Orthopedics Corp, other from Tissue Gene, outside the submitted work. R.E.D. reports other from Flexion Therapeutics, other from Orthofix Inc, other from Stryker Corp, other from United Orthopedics Corp, other from Tissue Gene, outside the submitted work. F.J.P. reports other from Biocomposites Inc., personal fees from Total Joint Orthopedics, personal fees and other from VisualDx, outside the submitted work.

\section{References}

1 Peersman G, Laskin R, Davis J, Peterson M. Infection in total knee replacement: a retrospective review of 6489 total knee replacements. Clin Orthop Relat Res 2001;(392):15-23

2 Phillips JE, Crane TP, Noy M, Elliott TSJ, Grimer RJ. The incidence of deep prosthetic infections in a specialist orthopaedic hospital: a 15-year prospective survey. J Bone Joint Surg Br 2006;88(07): 943-948

3 Pulido L, Ghanem E, Joshi A, Purtill JJ, Parvizi J. Periprosthetic joint infection: the incidence, timing, and predisposing factors. Clin Orthop Relat Res 2008;466(07):1710-1715

4 Pivec R, Naziri Q Issa K, Banerjee S, Mont MA. Systematic review comparing static and articulating spacers used for revision of infected total knee arthroplasty. J Arthroplasty 2014;29(03): 553-7.e1

5 Stammers J, Kahane S, Ranawat V, et al. Outcomes of infected revision knee arthroplasty managed by two-stage revision in a tertiary referral centre. Knee 2015;22(01):56-62

6 Mortazavi SMJ, Schwartzenberger J, Austin MS, Purtill JJ, Parvizi J. Revision total knee arthroplasty infection: incidence and predictors. Clin Orthop Relat Res 2010;468(08):2052-2059
7 Silvestre A, Almeida F, Renovell P, Morante E, López R. Revision of infected total knee arthroplasty: two-stage reimplantation using an antibiotic-impregnated static spacer. Clin Orthop Surg 2013;5 (03):180-187

8 Tigani D, Trisolino G, Fosco M, Ben Ayad R, Costigliola P. Two-stage reimplantation for periprosthetic knee infection: influence of host health status and infecting microorganism. Knee 2013;20 (01):9-18

9 Calton TF, Fehring TK, Griffin WL. Bone loss associated with the use of spacer blocks in infected total knee arthroplasty. Clin. Orthop. Relat. Res. 1997;LLC:148-154

10 Fehring KA, Abdel MP, Ollivier M, Mabry TM, Hanssen AD. Repeat two-stage exchange arthroplasty for periprosthetic knee infection is dependent on host grade. J Bone Joint Surg Am 2017;99 (01):19-24

11 Romanò CL, Gala L, Logoluso N, Romanò D, Drago L. Two-stage revision of septic knee prosthesis with articulating knee spacers yields better infection eradication rate than one-stage or twostage revision with static spacers. Knee Surg Sports Traumatol Arthrosc 2012;20(12):2445-2453

12 Johnson AJ, Sayeed SA, Naziri Q, Khanuja HS, Mont MA. Minimizing dynamic knee spacer complications in infected revision arthroplasty. Clin Orthop Relat Res 2012;470(01): 220-227

13 Mazzucchelli L, Rosso F, Marmotti A, Bonasia DE, Bruzzone M, Rossi R. The use of spacers (static and mobile) in infection knee arthroplasty. Curr Rev Musculoskelet Med 2015;8(04): 373-382

14 Park S-J, Song E-K, Seon J-K, Yoon T-R, Park G-H. Comparison of static and mobile antibiotic-impregnated cement spacers for the treatment of infected total knee arthroplasty. Int Orthop 2010;34 (08):1181-1186

15 Hsu YC, Cheng HC, Ng TP, Chiu KY. Antibiotic-loaded cement articulating spacer for 2-stage reimplantation in infected total knee arthroplasty: a simple and economic method. J Arthroplasty 2007;22(07):1060-1066

16 Fehring TK, Odum S, Calton TF, Mason JB. Articulating versus static spacers in revision total knee arthroplasty for sepsis. The Ranawat Award. Clin Orthop Relat Res 2000;(380):9-16

17 Emerson RH Jr, Muncie M, Tarbox TR, Higgins LL. Comparison of a static with a mobile spacer in total knee infection. Clin Orthop Relat Res 2002;(404):132-138

18 Wood JH, Conway JD. Advanced concepts in knee arthrodesis. World J Orthop 2015;6(02):202-210

19 Wiedel JD. Salvage of infected total knee fusion: the last option. Clin Orthop Relat Res 2002;(404):139-142

20 Waldman BJ, Mont MA, Payman KR, et al. Infected total knee arthroplasty treated with arthrodesis using a modular nail. Clin Orthop Relat Res 1999;(367):230-237

21 Bargiotas K, Wohlrab D, Sewecke JJ, Lavinge G, DeMeo PJ, Sotereanos NG. Arthrodesis of the knee with a long intramedullary nail following the failure of a total knee arthroplasty as the result of infection. Surgical technique. J Bone Joint Surg Am 2007;89(Suppl 2 Pt.1):103-110

22 Mohamed NS, Etcheson JI, Wilkie WA, et al. Two-stage exchange using a short intramedullary nail for treatment of periprosthetic knee infections: a technique worth questioning. J Knee Surg 2020 (ePub ahead of print). Doi: 10.1055/s-0040-1708856

23 Friedrich MJ, Schmolders J, Wimmer MD, et al. Two-stage knee arthrodesis with a modular intramedullary nail due to septic failure of revision total knee arthroplasty with extensor mechanism deficiency. Knee 2017;24(05): 1240-1246

24 Gathen M, Wimmer MD, Ploeger MM, et al. Comparison of twostage revision arthroplasty and intramedullary arthrodesis in patients with failed infected knee arthroplasty. Arch Orthop Trauma Surg 2018;138(10):1443-1452 
46 Treatment of PJI in Total Knee Arthroplasty with a Temporary Intramedullary Nail Mohamed et al.

25 Qiu YY, Yan CH, Chiu KY, Ng FY. Review article: bone defect classifications in revision total knee arthroplasty. J Orthop Surg (Hong Kong) 2011;19(02):238-243

26 McPherson EJ, Woodson C, Holtom P, Roidis N, Shufelt C, Patzakis M. Periprosthetic total hip infection: outcomes using a staging system. Clin Orthop Relat Res 2002;(403):8-15

27 Diaz-Ledezma C, Higuera CA, Parvizi J. Success after treatment of periprosthetic joint infection: a Delphi-based international multidisciplinary consensus. Clin Orthop Relat Res 2013;471(07): 2374-2382

28 Kubista B, Hartzler RU, Wood CM, Osmon DR, Hanssen AD, Lewallen DG. Reinfection after two-stage revision for periprosthetic infection of total knee arthroplasty. Int Orthop 2012;36(01):65-71
29 Choi H-R, Malchau H, Bedair H. Are prosthetic spacers safe to use in 2-stage treatment for infected total knee arthroplasty? J Arthroplasty 2012;27(08):1474-1479.e1

30 Lichstein P, Su S, Hedlund $\mathrm{H}$, et al. Treatment of periprosthetic knee infection with a two-stage protocol using static spacers. Clin Orthop Relat Res 2016;474(01):120-125

31 Freeman MG, Fehring TK, Odum SM, Fehring K, Griffin WL, Mason JB. Functional advantage of articulating versus static spacers in 2stage revision for total knee arthroplasty infection. J Arthroplasty 2007;22(08):1116-1121

32 Jämsen E, Sheng P, Halonen P, et al. Spacer prostheses in two-stage revision of infected knee arthroplasty. Int Orthop 2006;30(04): 257-261 